\title{
Serum miR-210-3p can be used to differentiate between patients with pancreatic ductal adenocarcinoma and chronic pancreatitis
}

\author{
MAŁGORZATA GUZ ${ }^{1}$, WITOLD JELENIEWICZ ${ }^{1}$, MAREK CYBULSKI ${ }^{1}$, \\ JOANNA KOZICKA ${ }^{2}$, JACEK KURZEPA ${ }^{3}$ and AGNIESZKA MĄDRO ${ }^{2}$ \\ ${ }^{1}$ Department of Biochemistry and Molecular Biology, Medical University of Lublin, 20-093 Lublin; \\ ${ }^{2}$ Department of Gastroenterology with Endoscopic Unit, Medical University of Lublin, 20-954 Lublin; \\ ${ }^{3}$ Department of Medical Chemistry, Medical University of Lublin, 20-093 Lublin, Poland
}

Received July 2, 2020; Accepted October 13, 2020

DOI: $10.3892 /$ br.2020.1386

\begin{abstract}
Patients with chronic pancreatitis (CP) are at risk of developing pancreatic ductal adenocarcinoma (PDAC). To the best of our knowledge, there are no suitable non-invasive biomarkers for differentiation between $\mathrm{CP}$ and PDAC; however, potential molecular candidates include circulating miRNAs due to ease of extraction, their stability and tissue specificity. Therefore, the aim of the present study was to identify potential serum marker(s) that may be used for differentiating between CP and PDAC. In total, 77 patients were enrolled in the present study; 34 patients with $\mathrm{CP}, 26$ patients with $\mathrm{PDAC}$ and a control group of 17 healthy individuals. Expression of miR-10b-5p, miR-106b-5p, miR-210-3p and miR-216a-5p in serum was determined by reverse transcription-quantitative PCR. Serum miRNA expression levels in patients with CP, PDAC and in the control group were compared. Routine biochemical blood parameters were determined and correlation analysis of these parameters with miRNA expression was performed. Expression of miR-210-3p was increased in the sera of patients with PDAC compared with the CP patients $(\mathrm{P}=0.015)$ and with the control group $(\mathrm{P}<0.001)$. MiR-106b-5p $(\mathrm{P}=0.056)$ and miR-10b-5p $(\mathrm{P}=0.080)$ were not significantly upregulated in patients with PDAC compared with those with CP. Analysis of miRNA expression in relation to laboratory blood parameters showed positive correlations between miR-210-3p with alkaline phosphatase $(\mathrm{r}=0.605 ; \mathrm{P}=0.022)$ and with $\gamma$-glutamyltranspeptidase $(\mathrm{r}=0.529 ; \mathrm{P}=0.029)$ in PDAC. The novel finding of the present study was that miR-10b-5p was positively correlated with $C$-reactive protein $(r=0.429$; $\mathrm{P}=0.047)$ in patients with PDAC and with carbohydrate antigen 19-9 ( $\mathrm{r}=0.483 ; \mathrm{P}=0.005)$ in $\mathrm{CP}$. Based on the preliminary data
\end{abstract}

Correspondence to: Dr Małgorzata Guz, Department of Biochemistry and Molecular Biology, Medical University of Lublin, ul. Chodźki 1, 20-093 Lublin, Poland

E-mail: malgorzata.guz@umlub.pl

Key words: serum microRNA, diagnostic tool, cancer, inflammation of the pancreas, pancreatic cancer, chronic pancreatitis obtained in the present study, it was concluded that miR-210-3p may be used as a non-invasive biomarker that can be used to distinguish between patients with PDAC and CP.

\section{Introduction}

Chronic pancreatitis (CP) is a type of pathologic fibroinflammatory syndrome of the exocrine pancreas, with genetic, environmental and/or other risk factors, and patients develop persistent responses to parenchymal injury and stress (1). In $\sim 70 \%$ of tumors of the pancreas, the tumor is primarily located within the head of the pancreas (2), and $~ 5 \%$ of patients with CP develop pancreatic cancer over the following 20 years (3). Pancreatic ductal adenocarcinoma (PDAC) is a common type of cancer of the pancreas with a high mortality rate, but does not have clearly defined symptoms (4). In $>50 \%$ of pancreatic cancer cases, patients are diagnosed with advanced stage cancer, which has the lowest 5-year relative survival rate (9\% for all stages) amongst all types of cancer (5). In $90 \%$ of patients with PDAC, the development of the cancer is sporadic, whereas in the other $10 \%$ of cases, there is a hereditary element (6). Only $15 \%$ of patients with PDAC are diagnosed at an early stage with an indication for surgical treatment, and at present, this is the only established curative means.

The majority of studies focused on differentiating between $\mathrm{CP}$ and PDAC primarily include evaluation of basic parameters, such as imaging, and clinical and biochemical markers. Carbohydrate antigen 19-9 (CA 19-9) is the most widely used biomarker for the diagnosis of pancreatic cancer in symptomatic patients (7). Unfortunately, it is not specific for this pathology, as CA 19-9 concentration in the blood may increase in other diseases, such as cirrhosis of the liver, cholangitis, CP and other tumors of the gastrointestinal tract (8). In addition, $5-10 \%$ of the population with a rare Lewis antigen system do not synthesize CA 19-9 (7). Patients with elevated levels of CA 19-9 are subjected to abdominal ultrasonography (US), computed tomography (CT), magnetic resonance imaging (MRI) or endoscopic ultrasound-guided fine-needle aspiration biopsy. The research is performed with the hope that it will allow for distinguishing between benign and neoplastic lesions, but these techniques do not always precisely define the type of pathology and are most often performed too late, 
and thus only confirm the fatal diagnosis (9). In addition, the symptoms of PDAC and CP are similar: Pain in the upper abdomen, weight loss, nausea and occasionally, jaundice. Both groups of patients often have a history of smoking and alcohol abuse. Novel diagnostic tests are necessary to differentiate PDAC from CP from which surgical and radical interventions, including pancreatoduodenectomy, can be avoided if not needed, as such treatments are associated with a large number of severe complications $(10,11)$.

To the best of our knowledge, there are no studies which have identified non-invasive methods for distinguishing $\mathrm{CP}$ from PDAC. Promising molecular candidates include short, non-coding RNAs, such as microRNAs (miRs/miRNAs), which are involved in regulation of key processes in all types of living cells, including cellular differentiation, apoptosis and growth (9). MiRNA expression profiles are tissue-specific, and aberrant miRNA expression can be indicative of pathologies such as inflammation or cancer (12). Based on the literature search performed for this study, four miRNAs: miR-10b-5p, miR-106b-5p, miR-210-3p and miR-216a-5p were selected, and their expression in the blood collected from patients with suspected CP and PDAC were compared to allow distinguishment between these two types of pathologies (13-17).

\section{Materials and methods}

Patients. A total of 77 patients were enrolled in the present study at the Department and Clinic of Gastroenterology with Endoscopic Unit, Medical University of Lublin. The study conformed with the principles outlined in the Declaration of Helsinki (18) and was approved by the Research Ethics Committee of the Medical University of Lublin (approval no. KE 0254-/54/2015). All participants provided written informed consent prior to enrolment in the study. The patients were divided into three groups: Group I consisted of 26 patients (17 males and 9 females; age range, 47-89, median age 58 years old) who were diagnosed with PDAC without a history of CP; Group II consisted of 34 patients with CP (27 males and 7 females; age range, 21-78, median age 39.5 years old); and Group III which consisted of 17 healthy patients (13 males, 4 females; age range, 22-50, median age 34 years old) and served as a control group according to imaging tests (CT, US) that excluded the presence of PDAC and $\mathrm{CP}$, as well as any other acute and chronic inflammation illnesses, verified by serum C-reactive protein (CRP) concentration measurement. The patients' characteristics are presented in Table I.

Blood sample collection. For routine blood sample analysis, $\sim 5 \mathrm{ml}$ of venous blood was collected into biochemical tubes without anticoagulant. After the blood had clotted, it was centrifuged at 2,500 $\mathrm{x}$ g for $10 \mathrm{~min}$ at $4^{\circ} \mathrm{C}$ twice to remove insoluble residues, then the supernatant was aliquoted in RNase-free tubes and stored at $-80^{\circ} \mathrm{C}$ until required for RNA isolation. The activity of lipase, amylase, alanine aminotransferase, aspartate aminotransferase, alkaline phosphatase (ALP), $\gamma$-glutamyltranspeptidase (GGTP), as well as hemoglobin (Hb), bilirubin, CRP and CA19-9 levels were determined by routine laboratory methods in The Central Laboratory (Independent Public Clinical Hospital No. 4, Lublin, Poland).
RNA isolation and cDNA synthesis. Total RNA, including miRNAs was isolated from serum using a miRCURY RNA Isolation kit according to the manufacturer's protocol (Biofluids; Exiqon; Qiagen, AB). Spike-ins UniSp 2, UniSp 4 and UniSp 5 were mixed with MS2 bacteriophage RNA (Roche Diagnostics) and were added to each sample to monitor RNA isolation. After optimization, the cDNA was synthesized from $4 \mu 1$ isolated RNA using a Universal cDNA Synthesis kit II according to the manufacturer's protocol (Exiqon; Qiagen, AB). Moreover, UniSp6 was used to monitor the quality of the reverse transcription reaction. For the negative controls, three reactions were prepared using the following reaction mixtures-without reverse transcriptase, without RNA template and using MS2 bacteriophage RNA as template.

Quantitative $(q) P C R$. Each cDNA sample was diluted with nuclease-free water and $4 \mu \mathrm{l}$ cDNA was mixed with $5 \mu 1$ SYBR-Green MasterMix (Exiqon; Qiagen, AB), and $1 \mu \mathrm{l}$ $\mathrm{LNA}^{\mathrm{TM}}$ primers (Exiqon; Qiagen, $\mathrm{AB}$ ). The final volume of the reaction mixture was $10 \mu \mathrm{l}$ and each reaction was carried out in triplicate. Amplification with real-time fluorescence detection was performed using a LightCycler ${ }^{\circledR} 480$ II instrument (Roche Applied Science) as follows; Initial denaturation, $10 \mathrm{~min}$ at $95^{\circ} \mathrm{C}$; followed by 45 cycles of $10 \mathrm{sec}$ at $95^{\circ} \mathrm{C}$ and $1 \mathrm{~min}$ at $60^{\circ} \mathrm{C}$. The degree of hemolysis of serum samples was evaluated using primers for miR-23a-3p and miR-451a. Differences between the miR-23a-3p and miR-451a $\mathrm{Cp}$ values $<5$ were considered hemolysis-free and such serum samples were further analyzed.

In accordance with recommendations from Exiqon, miR-30c, miR-103a-3p, miR-124-3p, miR-191-5p and miR-423-3p were considered as potential reference genes, amongst which, miR-103a-3p was selected for further analysis as it had the lowest degree of variation between analyzed groups $(\mathrm{P}>0.05)$. The relative expression of miRNAs was calculated using efficiency method with the LightCycler ${ }^{\circledR} 480$ SW version 1.5 software (Roche Diagnostics) according to Roche Operator's manual.

The sequences of the microRNA LNA ${ }^{\mathrm{TM}}$ primers (Exiqon; Qiagen, AB) used in the present study are: hsa-miR-30c-5p, UGUAAACAUCCUACACUCUCAGC; hsa-miR-103a-3p, AGCAGCAUUGUACAGGGCUAUGA; hsa-miR-124-3p, UAAGGCACGCGGUGAAUGCC; hsa-miR-191-5p, CAA CGGAAUCCCAAAAGCAGCUG; and hsa-miR-423-3p, AGC UCGGUCUGAGGCCCCUCAGU. For evaluation of the level of hemolysis target miRNA sequences were: miR-23a-3p, AUC ACAUUGCCAGGGAUUUCC; and hsa-miR-451a, AAACCG UUACCAUUACUGAGUU. Target miRNA sequences for potential miRNA biomarkers in differentiation of PDAC from CP were: hsa-miR-10b-5p, UACCCUGUAGAACCGAAU UUGUG; hsa-miR-106b-5p, UAA AGUGCUGACAGUGCA GAU; hsa-miR-210-3p, CUGUGCGUGUGACAGCGGCUGA; and hsa-miR-216a-5p, UAAUCUCAGCUGGCAACUGUGA. Spike-in target sequences used for RNA isolation control were: UniSp2, GUACUCGGCUUACGAUCGUAA; UniSp 4, GAU GGCAUUCGAUCAGUUCUA; and UniSp 5, GAUGCUACG GUCAAUGUCUAAG; for cDNA synthesis control, the UniSp 6 target sequence was CUAGUCCGAUCUAAGUCUUCGA.

Statistical analysis. Normality of distribution of miRNA expression was assessed using histograms and a Kolmogorov-Smirnov 
Table I. Clinicopathological characteristics of the recruited patients.

\begin{tabular}{|c|c|c|c|c|}
\hline $\begin{array}{l}\text { Clinicopathological } \\
\text { characteristic }\end{array}$ & $\begin{array}{c}\text { Chronic } \\
\text { pancreatitis group }\end{array}$ & $\begin{array}{c}\text { Pancreatic ductal } \\
\text { adenocarcinoma group }\end{array}$ & Control group & P-value \\
\hline \multicolumn{5}{|l|}{ Sex, $\mathrm{n}(\%)^{\mathrm{c}}$} \\
\hline Female & $7(20.59)$ & $9(34.61)$ & $4(23.53)$ & \multirow[t]{2}{*}{0.455} \\
\hline Male & $27(79.41)$ & $17(65.38)$ & $13(76.47)$ & \\
\hline Median age, years (range) ${ }^{\mathrm{d}}$ & $39.5(21-78)$ & $58.0(47-89)$ & $34.0(22-50)$ & $0.001^{\mathrm{b}}$ \\
\hline \multicolumn{5}{|l|}{ Smoking status, $\mathrm{n}(\%)^{\mathrm{a}}$} \\
\hline Smokers & $30(88.23)$ & $20(76.92)$ & $9(52.94)$ & \multirow[t]{2}{*}{$0.019^{\mathrm{a}}$} \\
\hline Non-smokers & 4 (11.76) & $6(23.08)$ & $8(47.06)$ & \\
\hline History of alcohol abuse, $\mathrm{n}(\%)^{\mathrm{c}}$ & $31(91.18)$ & $17(65.38)$ & $5(29.41)$ & $0.001^{\mathrm{b}}$ \\
\hline Diabetes $\mathrm{n}(\%)^{\mathrm{c}}$ & $14(41.18)$ & $6(23.08)$ & $2(11.76)$ & 0.068 \\
\hline \multicolumn{5}{|l|}{ Stage, $\mathrm{n}(\%)$} \\
\hline Early stage & - & $4(15.38)$ & - & - \\
\hline Locally advanced stage & - & $12(46.15)$ & - & - \\
\hline Advanced stage & - & $10(38.46)$ & - & - \\
\hline
\end{tabular}

${ }^{\mathrm{a}} \mathrm{P}<0.05 ;{ }^{\mathrm{b}} \mathrm{P}<0.01 ;{ }^{\mathrm{c}} \chi^{2}$-test; ${ }^{\mathrm{d}}$ Kruskal-Wallis test.

or Shapiro-Wilk tests. Since the distribution was not normal, differences in miRNA expression amongst the three groups (CP, PDAC and the control group) were analyzed using the non-parametric Kruskal-Wallis ANOVA by ranks and Mann Whitney U tests. Correlations between variables were analyzed using a Spearman-rank correlation coefficient test. Differences in frequencies were compared using a $\chi^{2}$ test. $\mathrm{P}<0.05$ was considered to indicate a statistically significant difference. Statistical analysis was performed using Statistica, version 13.3 (TIBCO Software Inc.).

\section{Results}

Expression of miR-10b-5p was observed in 24/26 patients with PDAC (92.3\%), 22/34 patients with CP (64.7\%) and in $17 / 17$ individuals in the control group (100\%). Expression of miR-106b-5p was observed in $25 / 26$ patients with PDAC (96.1\%), 31/34 patients with CP (91.7\%), and in $17 / 17$ individuals in the control group (100\%). Expression of miR-210-3p was observed in $22 / 26$ patients with PDAC (84.6\%), 21/34 patients with CP (61.8\%), and 16/17 patients in the control group (94.1\%). Expression of miR-216a-5p was observed in only two of the groups; in 5/26 patients with PDAC (19.2\%) and in 8/34 patients with CP (20.6\%). The percentage of samples expressing each miRNA assessed is shown in Fig. 1.

Serum miRNA levels were compared in the patients with CP, PDAC and the control group. Significantly higher expression levels of miR-210-3p were observed in the patients with $\mathrm{CP}$ and PDAC compared with the control group ( $\mathrm{P}=0.0002$; Fig. 2).

Comparative analysis showed significantly higher expression levels of miR-210-3p in the patients with PDAC compared with the patients with $\mathrm{CP}(\mathrm{P}=0.015)$, whereas expression of miR-106b-5p and miR-10b-5p tended to be higher in the patients with PDAC compared with those with $\mathrm{CP}$, although

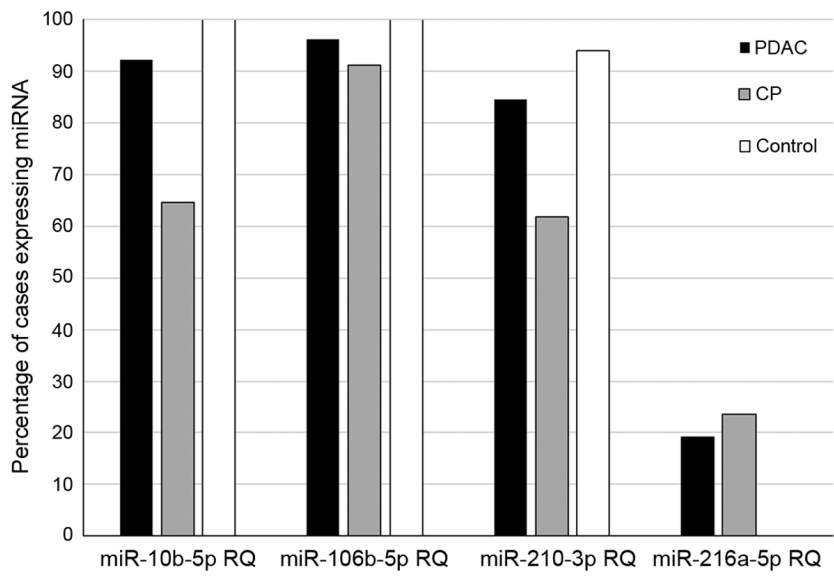

Figure 1. Graph representing the percentage of samples expressing each analyzed miRNA in patients with PDAC and CP, as well as the control group. $\mathrm{RQ}$, relative expression; miRNA/miR, microRNA; $\mathrm{CP}$, chronic pancreatitis; PDAC, pancreatic ductal adenocarcinoma.

the difference was not significant $(\mathrm{P}=0.056$ and $\mathrm{P}=0.080$, respectively). When analyzing miRNA expression in the PDAC group in comparison with the control group, only miR-210-3p expression in PDAC patients was significantly increased $(\mathrm{P}<0.001)$, and additionally its expression was correlated with ALP activity $(\mathrm{r}=0.605, \mathrm{P}=0.022)$, as well as with GGTP activity $(\mathrm{r}=0.529, \mathrm{P}=0.029)$.

Of minor clinical importance there was a statistically significant correlation between miR-10b-5p and the concentration of CRP $(\mathrm{r}=0.429, \mathrm{P}=0.047)$ in the PDAC group, and with CA 19-9 content $(\mathrm{r}=0.483, \mathrm{P}=0.005)$ in the $\mathrm{CP}$ group. All four statistically significant correlations between expression of miRNA and basic clinical parameters in PDAC and CP are shown in Table II. There were no other statistically significant correlations identified $(\mathrm{P}>0.05)$. Inter-correlations between selected miRNAs within analyzed patient groups were also 
Table II. Correlation between the assessed miRNAs and clinical parameters in patients with PDAC and CP.

\begin{tabular}{lccc}
\hline A, PDAC & & & \\
\hline Clinical parameter & Relative expression of the miRNA & $\mathrm{r}$ & P-value \\
\hline C-reactive protein & miR-10b-5p & 0.429 & $0.047^{\mathrm{a}}$ \\
Alkaline phosphatase & miR-210-3p & 0.605 & $0.022^{\mathrm{a}}$ \\
$\gamma$-glutamyltranspeptidase & miR-210-3p & 0.529 & $0.029^{\mathrm{a}}$ \\
\hline
\end{tabular}

$\mathrm{B}, \mathrm{CP}$

\begin{tabular}{lccc}
\hline Clinical parameter & Relative expression of the miRNA & r & P-value \\
\hline Carbohydrate antigen 19-9 & miR-10b-5p & 0.483 & $0.005^{\mathrm{b}}$ \\
\hline
\end{tabular}

${ }^{\mathrm{a}} \mathrm{P}<0.05,{ }^{\mathrm{b}} \mathrm{P}<0.01$. $\mathrm{CP}$, chronic pancreatitis; $\mathrm{PDAC}$, pancreatic ductal adenocarcinoma; miR/miRNA, microRNA.

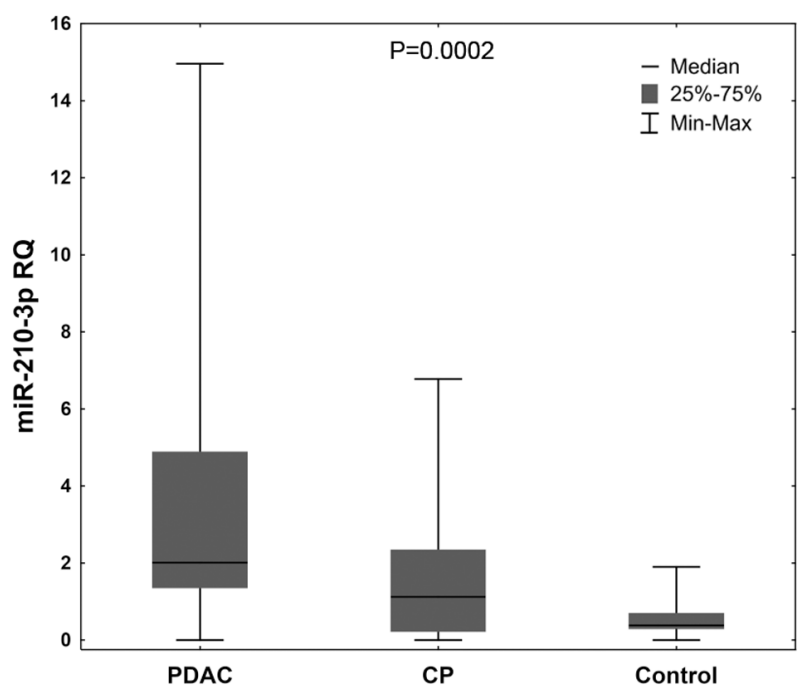

Figure 2. RQ of miR-210-3p in patients with PDAC and CP, as well as the control group. Data were analyzed using a Kruskal-Wallis test to determine whether a significant difference existed between the 3 groups. RQ, relative expression; miRNA/miR, microRNA; CP, chronic pancreatitis; PDAC, pancreatic ductal adenocarcinoma.

identified, but they were not statistically significant (data not shown).

\section{Discussion}

CP and PDAC are diseases with several similar symptoms. There is an inherent problem of distinguishing inflammation within the pancreas from neoplastic lesions, augmented by the lack of non-invasive diagnostic methods. Often, surgical interventions turn out to be an unnecessary risk that endangers a patient's health, therefore, discovery of blood-based markers is urgently required, which, together with determination of conventional clinical parameters, can enable a physician to distinguish between CP and PDAC. Complex networks formed by miRNAs regulate cellular development, differentiation and homeostasis (19). Alterations in the expression of miRNAs are associated with the number of diseases, including inflammatory diseases and cancer (20). In order to search for serum miRNAs that can be used to differentiate between CP and PDAC, candidate miRNAs associated with cancer and the inflammatory process were assessed; specifically miR-10b-5p, miR-106b-5p, miR-210-3p and miR-216a-5p, all of which have been previously found to be significantly upregulated in PDAC $(13,14,21,22)$. Amongst the selected miRNAs, miR-210-3p was deemed to be the most promising as a serum biomarker for use in differentiation between CP and PDAC, as its expression was higher in the patients with PDAC compared with those with $\mathrm{CP}$, and miR-106b-5p expression tended to be higher in patients with PDAC than those with CP. Studies using larger cohorts are thus required to determine/confirm the reliability of these miRs as markers to use to differentiate between these two pathologies. In $\sim 70 \%$ of cases of pancreatic cancer, the cancer is located within the pancreatic head and the majority of patients develop obstructive cholestasis resulting in increased ALP and GGTP serum levels (23). Significant positive correlations were identified between the expression of miR-210-3p and the activities of the cholestasis-related enzymes ALP and GGTP in patients with PDAC. This suggests that combined assessment of these parameters (miR-210-3p levels and ALP/GGTP activity) may improve the accuracy of early diagnosis and may provide a non-invasive diagnostic tool for distinguishing between PDAC $\mathrm{CP}$ in the future. There was a significant correlation between miR-10b-5p and CRP levels and CA 19-9 content. To date, there are no studies that have shown an association between expression of miR-10b-5p and CP, to the best of our knowledge. The role of miR-10b-5p in the course of carcinogenesis has already been described (24). Moreover, recently published data has shown that miR-10b may serve as a candidate predictive marker, as its expression is associated with significantly improved overall survival ( 2 months) in PDAC patients treated with a combination of gemcitabine and Galunisertib (a transforming growth factor $\beta$ receptor I inhibitor) $(25,26)$.

The majority of published studies have focused on comparative analysis of miR-210 expression in the serum, tissue specimen or pancreatic juice of patients with PDAC in relation to individuals with non-pancreatic diseases or healthy 
subjects (27-29). In the present study, significantly higher levels of miR-210-3p were observed in the sera of patients with PDAC compared with the healthy individuals, which confirms reports on the involvement of miR-210 in the regulation of expression of a number of genes associated with malignant transformation. The oncogenic effects of miR-210 have been described in pancreatic, prostate, ovary, bladder, breast and bone cancer $(15,28,30-33)$. Development of hypoxia in the most common pancreatic cancer, adenocarcinoma, results in a poor response to radiotherapy and chemotherapy (34). A state of oxygen deficiency in tissues induces the expression of specific miRNAs, including miR-210-3p, which is termed hypoxamiR (16,35). Genes regulated by miR-210 are involved in cell division and migration, angiogenesis, mitochondrial metabolism, DNA repair or chromatin remodeling (36-38). Anaerobic environments stimulate activation of pancreatic stellate cells, angiogenesis and fibrosis (39-42). Angiogenesis underlies the repair process that is observed in the course of $\mathrm{CP}$, and is a mechanism that also enables the development and growth of cancer (43). Upregulated expression of miR-210-3p expression in cells is reflected by its high levels in the blood (28); experimentally confirmed higher serum levels in patients with PDAC indicate higher expression of this miRNA in the cells where the aforementioned molecular changes may have occurred.

Limitations of the present study include the variance in sample sizes and a relatively low number of enrolled participants, and thus, the results of the present pilot study should be validated using larger cohorts.

In conclusion, the role of miRNAs in the pathogenesis of $\mathrm{CP}$ and PDAC highlights their potential use as non-invasive markers for detection and diagnosis of these diseases. It is hypothesized that miR-210-3p may be a useful biomarker for differentiation between these two pancreatic diseases. Additionally, the correlation between miR-210-3p with biochemical parameters, such as the activity of GGTP and ALP, enhances its prognostic value.

\section{Acknowledgements}

We would like to thank Ms. Agnieszka Styczyńska (Department of Biochemistry and Molecular Biology at the Medical University of Lublin) for editorial assistance and for proofreading the manuscript.

\section{Funding}

This work was supported by The Polish Ministry of Science and Higher Education Grant (grant no. MNmb 401).

\section{Availability of data and materials}

The datasets used and/or analyzed during the present study are available from the corresponding author on reasonable request.

\section{Authors' contributions}

$\mathrm{AM}, \mathrm{MG}$ and $\mathrm{JKu}$ designed, planned and supervised the study. MG and AM wrote and revised the manuscript. MG and WJ performed the experiments. MC performed the statistical analysis. MG, WJ and MC interpreted the results.
Samples and clinical data were collected by JKo. All authors read and approved the final version of the manuscript.

\section{Ethics approval and consent to participate}

The present study was approved by the Research Ethics Committee of the Medical University of Lublin (approval no. KE 0254-/54/2015).

\section{Patients consent for publication}

All participants provided written informed consent prior to enrolment in the study.

\section{Competing interests}

The authors declare that they have no competing interests.

\section{References}

1. Whitcomb DC, Frulloni L, Garg P, Greer JB, Schneider A, Yadav D and Shimosegawa T: Chronic pancreatitis: An international draft consensus proposal for a new mechanistic definition. Pancreatology 16: 218-224, 2016.

2. Ansari D, Tingstedt B, Andersson B, Holmquist F, Sturesson C, Williamsson C, Sasor A, Borg D, Bauden M and Andersson R: Pancreatic cancer: Yesterday, today and tomorrow. Futur Oncol 12: 1929-1946, 2016.

3. Raimondi S,Lowenfels AB, Morselli-Labate AM, Maisonneuve P and Pezzilli R: Pancreatic cancer in chronic pancreatitis; aetiology, incidence, and early detection. Best Pract Res Clin Gastroenterol 24: 349-358, 2010

4. Wu DJ, Jiang YS, He RZ, Tao LY, Yang MW, Fu XL, Yang JY and Zhu K: High expression of WNT7A predicts poor prognosis and promote tumor metastasis in pancreatic ductal adenocarcinoma. Sci Rep 8: 15792, 2018.

5. Siegel RL, Miller KD and Jemal A: Cancer statistics, 2019. CA Cancer J Clin 69: 7-34, 2019

6. Becker AE, Hernandez YG, Frucht H and Lucas AL: Pancreatic ductal adenocarcinoma: Risk factors, screening, and early detection. World J Gastroenterol 20: 11182-11198, 2014.

7. Ballehaninna UK and Chamberlain RS: The clinical utility of serum CA 19-9 in the diagnosis, prognosis and management of pancreatic adenocarcinoma: An evidence based appraisal. J Gastrointest Oncol 3: 105-119, 2012.

8. Duffy MJ, Sturgeon C, Lamerz R, Haglund C, Holubec VL, Klapdor R, Nicolini A, Topolcan O and Heinemann V: Tumor markers in pancreatic cancer: A European group on tumor markers (EGTM) status report. Ann Oncol 21: 441-447, 2010.

9. Halkova T, Cuperkova R, Minarik $M$ and Benesova L: MicroRNAs in pancreatic cancer: Involvement in carcinogenesis and potential use for diagnosis and prognosis. Gastroenterol Res Pract 2015: 892903, 2015

10. Smith CD, Behrns KE, Van Heerden JA and Sarr MG: Radical pancreatoduodenectomy for misdiagnosed pancreatic mass. Br J Surg 81: 585-589, 1994.

11. van Gulik TM, Reeders JW, Bosma A, Moojen TM, Smits NJ, Allema JH, Rauws EA, Offerhaus GJ, Obertop H and Gouma DJ: Incidence and clinical findings of benign, inflammatory disease in patients resected for presumed pancreatic head cancer. Gastrointest Endosc 46: 417-423, 1997.

12. Nana-Sinkam SP, Fabbri $M$ and Croce CM: MicroRNAs in cancer: Personalizing diagnosis and therapy. Ann N Y Acad Sci 1210: 25-33, 2010.

13. Sheedy $\mathrm{P}$ and Medarova $\mathrm{Z}$ : The fundamental role of miR-10b in metastatic cancer. Am J Cancer Res 8: 1674-1688, 2018.

14. Yonemori K, Seki N, Idichi T, Kurahara H, Osako Y, Koshizuka K, Arai T, Okato A, Kita Y, Arigami T, et al: The microRNA expression signature of pancreatic ductal adenocarcinoma by RNA sequencing: Anti-tumour functions of the microRNA-216 cluster. Oncotarget 8: 70097-70115, 2017.

15. Ding L, Zhao L, Chen W, Liu T, Li Z and Li X: miR-210, a modulator of hypoxia-induced epithelial-mesenchymal transition in ovarian cancer cell. Int J Clin Exp Med 8: 2299-2307, 2015. 
16. Dang K and Myers KA: The role of hypoxia-induced miR-210 in cancer progression. Int J Mol Sci 16: 6353-6372, 2015.

17. Kaur S, Krishn SR, Rachagani S and Batra SK: Significance of microRNA-based biomarkers for pancreatic cancer. Ann Transl Med 3: 277, 2015.

18. World Medical Association: Declaration of Helsinki world medical association declaration of Helsinki. Ethical principles for medical research involving human subjects. Bull World Health Organ 79: 373-374, 2001.

19. Gebert LFR and MacRae IJ: Regulation of microRNA function in animals. Nat Rev Mol Cell Biol 20: 21-37, 2019.

20. Raisch J, Darfeuille-Michaud A and Nguyen HT: Role of microRNAs in the immune system, inflammation and cancer. World J Gastroenterol 19: 2985-2996, 2013.

21. Li Y and Sarkar FH: MicroRNA targeted therapeutic approach for pancreatic cancer. Int J Biol Sci 12: 326-337, 2016.

22. Vila-Casadesús M, Vila-Navarro E, Raimondi G, Fillat C, Castells A, Lozano JJ and Gironella M: Deciphering microRNA targets in pancreatic cancer using miRComb $\mathrm{R}$ package. Oncotarget 9: 6499-6517, 2018.

23. Vogel A, Kullmann F, Kunzmann V, Al-Batran SE, Oettle H, Plentz R, Siveke J, Springfeld C and Riess H: Patients with advanced pancreatic cancer and hyperbilirubinaemia: Review and German expert opinion on treatment with nab-paclitaxel plus gemcitabine. Oncol Res Treat 38: 596-603, 2015.

24. Ouyang H, Gore J, Deitz S and Korc M: microRNA-10b enhances pancreatic cancer cell invasion by suppressing TIP30 expression and promoting EGF and TGF- $\beta$ actions. Oncogene 33 : 4664-4674, 2014.

25. Melisi D, Garcia-Carbonero R, Macarulla T, Pezet D, Deplanque G, Fuchs M, Trojan J, Kozloff M, Simionato F, Cleverly A, et al: TGF $\beta$ receptor inhibitor galunisertib is linked to inflammation- and remodeling-related proteins in patients with pancreatic cancer. Cancer Chemother Pharmacol 83: 975-991, 2019.

26. Melisi D, Garcia-Carbonero R, Macarulla T, Pezet D, Deplanque G, Fuchs M, Trojan J, Oettle H, Kozloff M, Cleverly A, et al: Galunisertib plus gemcitabine vs. gemcitabine for first-line treatment of patients with unresectable pancreatic cancer. Br J Cancer 119: 1208-1214, 2018

27. Wang J, Raimondo M, Guha S, Chen J, Diao L, Dong X, Wallace MB, Killary AM, Frazier ML, Woodward TA, et al: Circulating microRNAs in pancreatic juice as candidate biomarkers of pancreatic cancer. J Cancer 5: 696-705, 2014

28. Ho AS, Huang X, Cao H, Christman-Skieller C, Bennewith $\mathrm{K}$, Le QT and Koong AC: Circulating miR-210 as a novel hypoxia marker in pancreatic cancer. Transl Oncol 3: 109-113, 2010.

29. Calatayud D, Dehlendorff C, Boisen MK, Hasselby JP, Schultz NA, Werner J, Immervoll H, Molven A, Hansen CP and Johansen JS: Tissue MicroRNA profiles as diagnostic and prognostic biomarkers in patients with resectable pancreatic ductal adenocarcinoma and periampullary cancers. Biomark Res 5: 8, 2017.

30. Cheng HH, Mitchell PS, KrohEM, Dowell AE, Chéry L, Siddiqui J, Nelson PS, Vessella RL, Knudsen BS, Chinnaiyan AM, et al Circulating microRNA profiling identifies a subset of metastatic prostate cancer patients with evidence of cancer-associated hypoxia. PLoS One 8: e69239, 2013.
31. Irlam-Jones JJ, Eustace A, Denley H, Choudhury A, Harris AL, Hoskin PJ and West CL: Expression of miR-210 in relation to other measures of hypoxia and prediction of benefit from hypoxia modification in patients with bladder cancer. Br J Cancer 115: 571-578, 2016.

32. Zhang H, Mai Q and Chen J: MicroRNA-210 is increased and it is required for dedifferentiation of osteosarcoma cell line. Cell Biol Int 41: 267-275, 2017.

33. Foekens JA, Sieuwerts AM, Smid M, Look MP, de Weerd V, Boersma AW, Klijn JG, Wiemer EA and Martens JW: Four miRNAs associated with aggressiveness of lymph node-negative, estrogen receptor-positive human breast cancer. Proc Natl Acad Sci USA 105: 13021-13026, 2008.

34. Koong AC, Mehta VK, Le QT, Fisher GA, Terris DJ, Brown JM, Bastidas AJ and Vierra M: Pancreatic tumors show high levels of hypoxia. Int J Radiat Oncol Biol Phys 48: 919-922, 2000.

35. Yan Y, Wang C, Zhou W, Shi Y, Guo P, Liu Y, Wang J, Zhang CY and Zhang C: Elevation of circulating miR-210-3p in high-altitude hypoxic environment. Front Physiol 7: 84, 2016.

36. Crosby ME, Kulshreshtha R, Ivan M and Glazer PM: MicroRNA regulation of DNA repair gene expression in hypoxic stress. Cancer Res 69: 1221-1229, 2009.

37. Mizuno Y, Tokuzawa Y,Ninomiya Y,YagiK, Yatsuka-Kanesaki Y, Suda T, Fukuda T, Katagiri T, Kondoh Y, Amemiya T, et al: miR-210 promotes osteoblastic differentiation through inhibition of AcvR1b. FEBS Lett 583: 2263-2268, 2009.

38. Chen Z, Li Y,Zhang H, Huang P and Luthra R: Hypoxia-regulated microRNA-210 modulates mitochondrial function and decreases ISCU and COX10 expression. Oncogene 29: 4362-4368, 2010.

39. Masamune A, Kikuta K, Watanabe T, Satoh K, Hirota M and Shimosegawa T: Hypoxia stimulates pancreatic stellate cells to induce fibrosis and angiogenesis in pancreatic cancer. Am J Physiol Gastrointest Liver Physiol 295: G709-G717, 2008.

40. Friess H, Yamanaka Y, Büchler M, Beger HG, Do DA, Kobrin MS and Korc M: Increased expression of acidic and basic fibroblast growth factors in chronic pancreatitis. Am J Pathol 144: 117-128, 1994.

41. Kobrin MS, Yamanaka Y, Friess H, Lopez ME and Korc M: Aberrant expression of type I fibroblast growth factor receptor in human pancreatic adenocarcinomas. Cancer Res 53: 4741-4744, 1993.

42. Yamanaka Y, Friess H, Buchler M, Beger HG, Uchida E, Onda M, Kobrin MS and Korc M: Overexpression of acidic and basic fibroblast growth factors in human pancreatic cancer correlates with advanced tumor stage. Cancer Res 53: 5289-5296, 1993.

43. Kuehn R, Lelkes PI, Bloechle C, Niendorf A and Izbicki JR: Angiogenesis, angiogenic growth factors, and cell adhesion molecules are upregulated in chronic pancreatic diseases: Angiogenesis in chronic pancreatitis and in pancreatic cancer. Pancreas 18: 96-103, 1999.

This work is licensed under a Creative Commons Attribution-NonCommercial-NoDerivatives 4.0 International (CC BY-NC-ND 4.0) License. 